\title{
New Approach for the Prediction of the Electric Field Distribution in Multimode Microwave-Heating Applicators With Mode Stirrers
}

\author{
Pedro Plaza-González, Juan Monzó-Cabrera, José M. Catalá-Civera, and David Sánchez-Hernández, Member, IEEE
}

\begin{abstract}
We present a new approach for inferring the electric field distribution inside materials in multimode cavities with mode stirrers. We calculate the electric field in the dielectric material by a two-dimensional modeling of a typical multimode microwave applicator with some mobile metallic sheets. We compare simulated results with classical approaches, such as Lambert's law or a constant electric field distribution. The proposed method allows for a better understanding of how these structures can be applied for heating materials when computing the microwave energy absorption in the dielectric. Finally, we perform experimental tests in a microwave multimode oven for validating purposes.
\end{abstract}

Index Terms-Dielectric materials, electromagnetic heating, finite-element analysis, mode stirrers.

\section{INTRODUCTION}

$\mathbf{M}$ ICROWAVE heating has been widely used in the industry as a way of accelerating heating processes of dielectric materials, in which conventional procedures are slow or inefficient. In this kind of application, the energy is introduced directly into the volume of the dielectric; thus, the quality of the process is highly dependent on the uniformity of the microwave electric field distribution along the material.

Static microwave applicator designs usually lead to nonuniform electric field patterns with undesirable hot temperature spots, with the heating process being more critical for low thermal conductivity materials. In order to achieve the desired uniformity, mode stirrers have been extensively used when multimode applicators are designed. Mode stirrers are mobile metallic elements that modify the electromagnetic (EM) boundary conditions within the microwave applicator, resulting in a temporal nonstationary electric field pattern over the material, which consequently improves heating uniformity [2]-[4].

For these multimode applicators, the explicit calculation of the electromagnetic field distribution, and thus the energy ab-

Manuscript received June 18, 2003; revised October 24, 2003. This work was supported in part by the Spanish Science and Technology Ministry under Project TIC2001-2778-CO2-02.

P. Plaza-González is with the ITACA Research Institute, Universidad Politécnica de Valencia, Valencia PO-46022, Spain (e-mail: pedplago@doctor.upv.es).

J. Monzó-Cabrera and D. Sánchez-Hernández are with the Departmento de Teoría de la Señal y Radiocomunicaciones, Universidad Politécnica de Cartagena, Cartagena PO-30202, Spain (e-mail: juan.monzo@upct.es; david. sanchez@upct.es).

J. M. Catalá-Civera is with the Departamento de Comunicaciones, Universidad Politécnica de Valencia, Valencia PO-46022, Spain (e-mail: jmcatala@dcom.upv.es).

Digital Object Identifier 10.1109/TMAG.2003.821560 sorption along the dielectric, has only been derived in the available literature, for empty or fully loaded canonical waveguides or cavities [5]. For partially loaded structures, like the problem under study, numerical techniques must be applied in order to obtain the spatial field distribution over the materials. Several numerical methods can be employed to solve Maxwell's equations in a multimode heating cavity, although the most popular techniques are based on the finite-element method (FEM) [6]-[10] and the finite-difference time-domain (FDTD) method [11]-[19]. Out of the two previously mentioned methods, the FEM represents a more agile technique since calculations can be performed for complex and arbitrary structures without the staircase meshing used in FDTD.

On the other hand, although mode stirrers are widely used by industrial applicators, the computation of the fields excited by mode stirrers in multimode cavities has not been extensively studied [20]. This has led to applicator designs based on the experience rather than on a deep comprehension of the electromagnetic problem. To overcome such difficulties, many authors either assume a simple constant electric field in the material or apply Lambert's law [1] to compute the field spatial distribution inside the sample being heated, rather than solving the exact formulation for the problem [21]-[23]. Although the use of such an approach is very popular for industrial applications, it may not be valid under certain situations, where the simplifications of the real problem can no longer be applied.

In this work, a new approach for determining the electric field inside materials in multimode cavities with laminate mode stirrers is presented. The proposed method allows for an improvement on the understanding of how these structures can be applied for heating dielectric materials. Electric field distributions on the materials are calculated and compared to those obtained with Lambert's law and with a constant electric field in order to establish the validity of these last approaches when used for inferring the spatial microwave energy absorption inside the dielectric. The influence of the geometry and the dielectric properties of the body being irradiated on the spatial field distribution are also discussed. Likewise, a set of measurements in a microwave multimode oven with some mobile plates has also been used to validate the simulated results.

\section{Mathematical Formulation of THE METHOD}

For the study of the electric field distributions in multimode applicators with mode stirrers, the schematic geometry depicted 


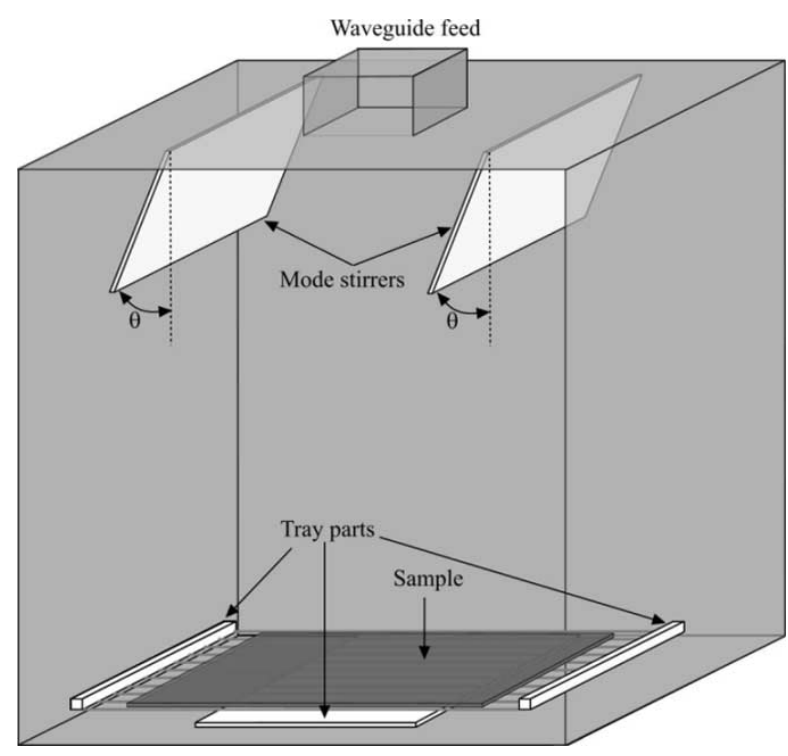

Fig. 1. General three-dimensional scheme of the multimode microwave applicator with mode stirrers and loaded with a dielectric sample.

in Fig. 1 was chosen. In Fig. 1, we can see the microwave applicator as a large metallic box with some metallic parts inside which are in continuous movement in order to establish nonuniform boundary conditions. The mathematical formulation associated with the study of these microwave structures is a boundary-value problem with sources in which the boundary conditions are changing with time.

However, if we assume that the heating rate of the processed material is slow compared with the movement of the stirrers, the position of the stirrers may be considered fixed at a given time slot and, consequently, the problem for this specific time slot can be solved in the frequency domain with the aid of the vector wave equation [5]

$$
\nabla^{2} \vec{E}+\omega^{2} \mu \varepsilon \vec{E}=0
$$

with $\vec{E}$ the vector electric field in the multimode cavity, $\omega$ the angular frequency, $\mu$ the permeability, and $\varepsilon$ the permittivity of the medium. In this study, we consider nonmagnetic materials characterized solely by its complex relative permittivity

$$
\varepsilon^{*}=\varepsilon^{\prime}-j \varepsilon^{\prime \prime}=\varepsilon^{\prime}-j \frac{\sigma}{\omega \varepsilon_{o}}
$$

where $\varepsilon^{\prime}$ is the relative dielectric constant, $\varepsilon^{\prime \prime}$ is the loss factor, $\varepsilon_{o}$ is the vacuum permittivity, and $\sigma$ is the conductivity of the material.

From the microwave electric field inside the cavity, the temperature evolution within the dielectric body is given by the so-called heat equation [1], [3] with internal heat generation

$$
\frac{\partial T}{\partial t}=\frac{k_{T}}{\rho \cdot c_{P}} \nabla^{2} T+\frac{Q_{\mathrm{gen}}}{\rho \cdot c_{P}}
$$

where $\rho\left(\mathrm{kg} / \mathrm{m}^{3}\right)$ represents the density of the body, $c_{\mathrm{p}}\left(\mathrm{J} / \mathrm{kg}^{\circ} \mathrm{C}\right)$ its specific heat and $k_{T}\left(\mathrm{~W} / \mathrm{m}^{\circ} \mathrm{C}\right)$ the thermal conductivity, $T\left({ }^{\circ} \mathrm{C}\right)$ is the temperature, and $t$ is the time (seconds). $Q_{\text {gen }}$
$\left(\mathrm{W} / \mathrm{m}^{3}\right)$ is known as the volumetric heat generation factor and takes into account the conversion of microwave energy into heat due to dielectric losses

$$
Q_{\text {gen }}=2 \pi f \varepsilon_{o} \varepsilon^{\prime \prime}\left|E_{\mathrm{rms}}\right|^{2}
$$

where $f$ is the frequency of the source and $E_{\text {rms }}$ is the effective electric field intensity in the material in volts per meter. This electric field, as can be derived from (1), depends on the boundary conditions of the microwave applicator and thus depends on the stirrer position. But, as mentioned above, if the speed of the stirrers is high enough (i.e., 100-200 cycles/min) in terms of the heating rate of the material, we can consider an electric field average of all the contributions of the different positions of the stirrers. Since in (4), $Q_{\text {gen }}$ is the average power that takes part in the heating of the dielectric, the use of the quadratic average of the instantaneous contributions of the electric field is proposed. Equation (5) shows the calculation of the average electric field in the sample $E_{\text {mean }}(x, y)$, obtained from a linear average of the absorbed power

$$
E_{\text {mean }}(x, y)=\sqrt{\frac{\left(\sum_{i}^{N} E_{i}^{2}(x, y)\right)}{N}}
$$

where $E_{i}(x, y)$ is the instantaneous spatial field distribution within the sample for position $i$ of the mode stirrers, and $N$ are the number of different simulated positions.

In order to solve the electric field distribution for each time slot, i.e., $E_{i}(x, y)$, the Helmholtz equation (1) has been solved by using the FEM replaced by its variational formulation, according to [24]

$$
\iint_{\Omega}\left(\frac{1}{\mu} \nabla E\right) \nabla T d \Omega+\omega^{2} \iint_{\Omega} \varepsilon E T d \Omega=0
$$

where $\Omega$ is the domain in which the problem is defined and $T$ are the so-called test functions. By adding the appropriate boundary conditions for the metallic walls and the source of the structure, and applying (6) to all the elements of discretization, this equation can be transformed into a linear system that can be readily solved. For this study, the Pdetool function included in Matlab was used in order to mesh the domain and to determine the electric field distribution inside the multimode cavity and the sample for each of the stirrers' positions. The Partial Differential Equation (PDE) Toolbox provides a Matlab integrated environment for the study and solution of partial differential equations in two space dimensions and time. The PDE Toolbox supplies several tools so that the user can define a PDE problem [definition of two-dimensional (2-D) regions, boundary conditions, and PDE coefficients], numerically discretize and solve the PDE equations, produce an approximation to the solution, and, finally, visualize the results [24].

The temperature in the dielectric material was calculated by the discretization of (3), which was numerically solved by employing an in-house code that uses the FDTD method. Since the 
electric field comes from a nonstructured FEM mesh, an irregular FDTD scheme has been accomplished to discretize the firstand second-order partial derivatives as

$$
\begin{aligned}
\frac{\partial^{2} T}{\partial x^{2}}= & \frac{1}{2}\left[\frac{1}{\Delta x_{i-1}^{2}}+\frac{1}{\Delta x_{i-1} \cdot \Delta x_{i-1}}\right] \cdot T_{i-1}^{l} \\
& -\frac{1}{2}\left[\frac{1}{\Delta x_{i-1}^{2}}+\frac{1}{\Delta x_{i}^{2}}+\frac{2}{\Delta x_{i} \Delta x_{i-1}}\right] \cdot T_{i}^{l} \\
& +\frac{1}{2}\left[\frac{1}{\Delta x_{i}^{2}}+\frac{1}{\Delta x_{i} \cdot \Delta x_{i-1}}\right] \cdot T_{i+1}^{l} \\
\frac{\partial T}{\partial x}= & \frac{T_{i+1}^{l}-T_{i}^{l}}{\Delta x_{i}} \\
\frac{\partial T}{\partial t}= & \frac{T_{i}^{l+1}-T_{i}^{l}}{\Delta t}
\end{aligned}
$$

where $\Delta x_{i}$ is the distance between consecutive nodes $i$ and $i+$ $1, T_{i}$ is the temperature of the node $i$, superscript $l$ represents the discretized time instant, and $\Delta t$ is the time step for each iteration. With this procedure, the temperature of the sample has been calculated as a function of the average electric field given by (4)-(6) and both the dielectric and thermal properties of the sample.

For the heat equation, an adiabatic boundary condition has been applied with

$$
\left.k_{T} \frac{\partial T}{\partial n}\right|_{S}=0
$$

where $n$ is the normal vector to the surface of the sample.

In order to compare this method to other more conventional approaches, Lambert's law, given by (11), will be used to compute the electric field spatial distribution in the material [22]

$$
\begin{aligned}
& \left|\vec{E}_{\text {Lambert }}\right| \\
& =\left|\vec{E}_{\text {surface }}\right|\left(e^{-\alpha x}+e^{-\alpha\left(x-L_{x}\right)}+e^{-\alpha y}+e^{-\alpha\left(y-L_{y}\right)}\right) .
\end{aligned}
$$

In (11), $E_{\text {surface }}$ is the electric field on the sample surface that best fits the behavior described by (1)-(6), $\alpha$ is the attenuation factor $(\mathrm{Np} / \mathrm{m})$ which depends upon the permittivity of the material [1], and $L_{x}$ and $L_{y}$ are the sample length in the canonical directions $x$ and $y$, respectively. An average constant electric field will also be used for comparison purposes and, additionally, to establish the limits in which it is possible to use this approximation. For this last purpose, a quadratic minimum error procedure will be applied to results given by (6) in order to get the best fitting curve of constant distribution to simulated results.

\section{NUMERICAL RESULTS}

By solving the equations described in the previous section, we can analyze the EM fields inside the microwave applicator as a function of the position of the mode stirrers. Fig. 2 illustrates a 2-D simplified scheme of the simulated cavity. From Fig. 2, one can observe the mode stirrers designed as two metallic sheets at the top of the applicator with a time-dependent position. The feeding port is situated at the upper wall of the multimode applicator and it consists of a WR-340 waveguide, which has been

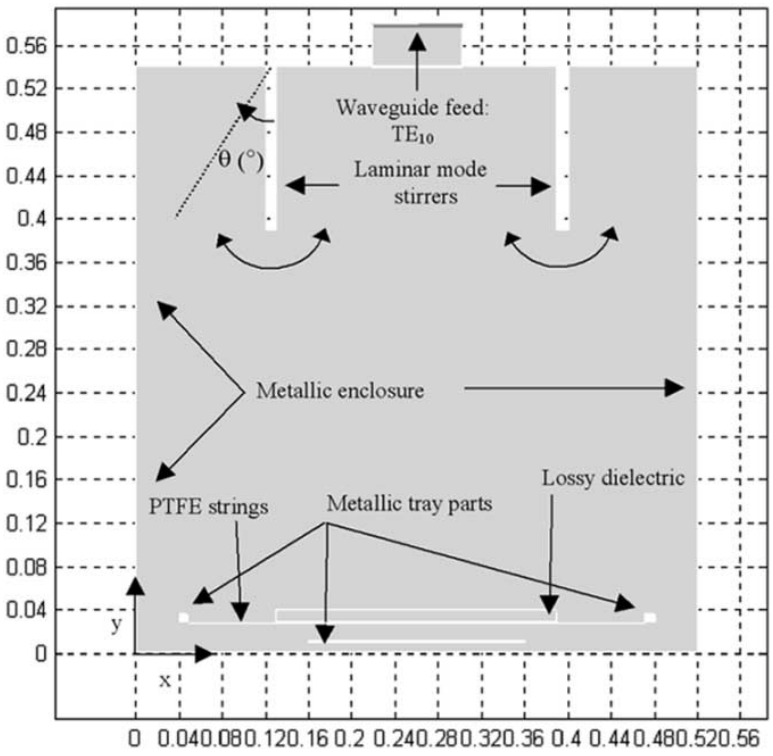

Fig. 2. 2-D simulation scheme for the multimode microwave cavity with laminate mode stirrers. Dielectric sample is placed over a PTFE holder at $y=0.035 \mathrm{~m}$ and from $x=0.13 \mathrm{~m}$ to $x=0.39 \mathrm{~m}$.

TABLE I

PARAMETERS USED IN SimUlation FOR FIGS. 3-8

\begin{tabular}{lll}
\hline \hline \multirow{2}{*}{ Symbol } & \multicolumn{1}{c}{ Quantity } & \multicolumn{1}{c}{ Value } \\
& & \multicolumn{1}{c}{$2.45 \mathrm{GHz}$} \\
$f$ & Frequency & $1450 \mathrm{~kg} / \mathrm{m}^{3}$ \\
$\rho$ & Sample density & $1250 \mathrm{~J} / \mathrm{kg}$ \\
$c_{p}$ & Sample specific heat & $0.8 \mathrm{~W} / \mathrm{m}^{\circ} \mathrm{C}$ \\
$k_{t}$ & Sample thermal conductivity & $1 \times 10^{3} \mathrm{~V} / \mathrm{m}$ \\
$E_{\text {source }}$ & TE 10 Electric field peak value & $2,15,30$ \\
$\varepsilon^{\prime}$ & Sample dielectric constant & $0.2,2.5,5$ \\
$\varepsilon^{\prime \prime}$ & Sample loss factor & From $45^{\circ}$ to $-45^{\circ}$ in $32 \mathrm{steps}$. \\
$\theta$ & Angle discretization & $0.26 \mathrm{~m}, \mathrm{x} \in[0.13,0.39] \mathrm{m}$ \\
$L$ & Sample Length & $2,5,10 \mathrm{~mm}$ \\
$t$ & Sample thickness & $y_{i n i}=0.035 \mathrm{~m}$ \\
& & $y_{\text {end }}=0.037,0.04,0.045 \mathrm{~m}$ \\
& & $x=0.52 \mathrm{~m}, y=0.54 \mathrm{~m}$ \\
& Multimode cavity dimensions & $x=0.01 \mathrm{~m}, y=0.15 \mathrm{~m}$ \\
\hline
\end{tabular}

excited with its dominant $\mathrm{TE}_{10}$ mode. Table I gives the parameters employed in the simulations, such as physical dimensions of the multimode cavity, dielectric and thermal properties of the material inside the cavity, and the positions considered for the movement of the mode stirrers.

Fig. 3 shows the electric field distribution in the cavity for four different positions of the metallic stirrers. From this figure, we can observe that the position of the mode stirrers inside the multimode applicator gives very different spatial field distributions in the cavity. It can be also appreciated by the gray levels in Fig. 3, that inside the sample the field strength is lower than in the cavity due to the energy reflected at the dielectric-air interface. Fig. 4 shows precisely the electric field distribution at the sample position for these different angles of the mode stirrer. These static distributions may correspond to those that we would have if no mode stirrers were installed in the applicator. Thus, as can be appreciated from the figures, the very different electric field profiles for the static situation would lead to unequal heating patterns. 

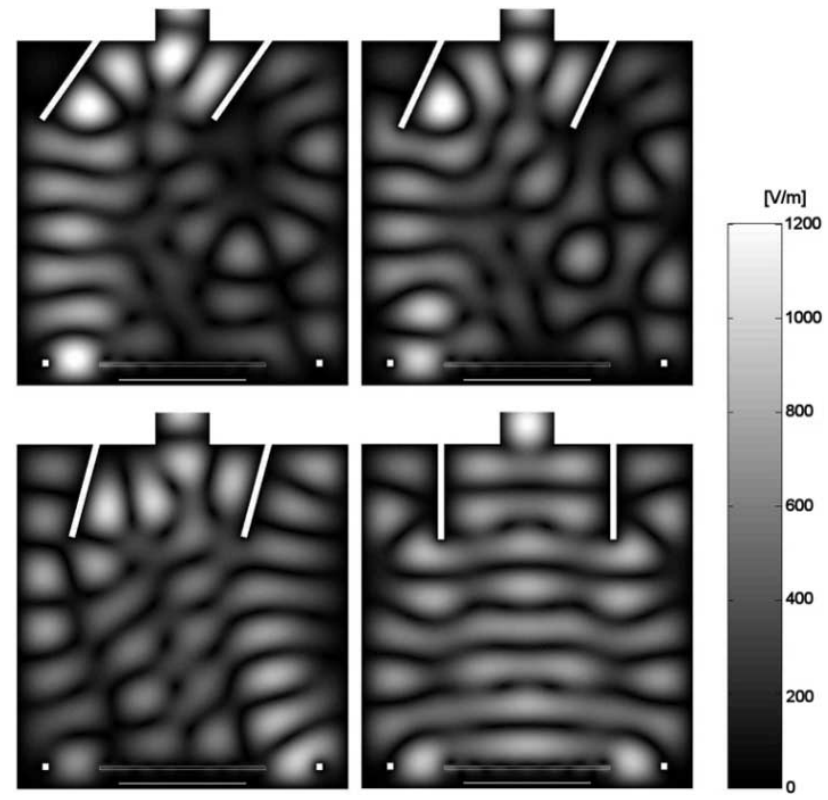

Fig. 3. Electric field distribution in the multimode cavity for four different mode stirrers with angle positions $\left(\theta=36^{\circ}, 25^{\circ}, 16^{\circ}, 1^{\circ}\right)$. Sample permittivity is $\varepsilon^{\prime}=30, \varepsilon^{\prime \prime}=5$, and sample thickness is $t=5 \mathrm{~mm}$.

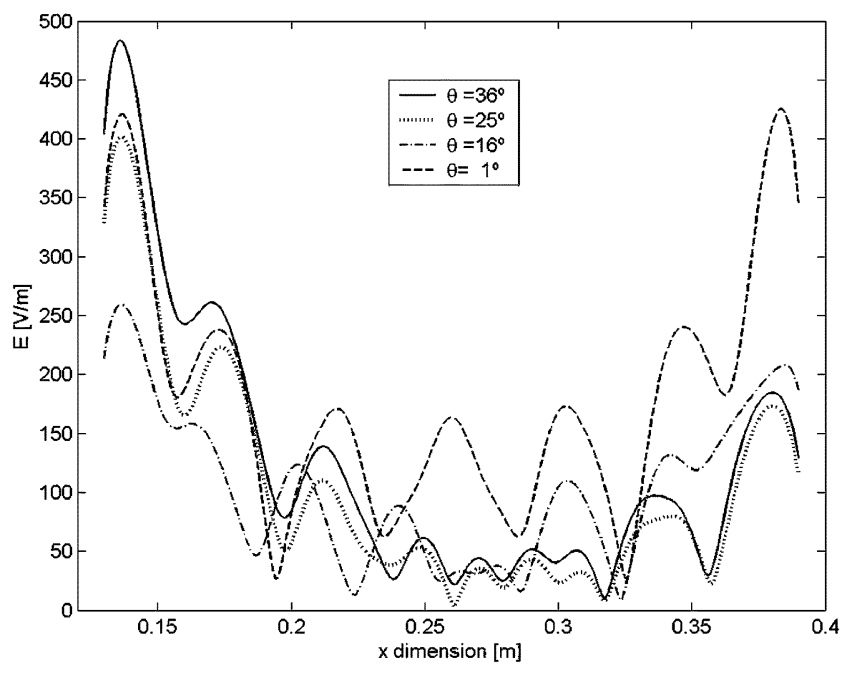

Fig. 4. Electric field distribution in the dielectric material for four different mode stirrers with angle positions $\left(\theta=36^{\circ}, 25^{\circ}, 16^{\circ}, 1^{\circ}\right)$. Sample permittivity is $\varepsilon^{\prime}=30, \varepsilon^{\prime \prime}=5$, and sample thickness is $t=5 \mathrm{~mm}$.

The method described above was used to understand the influence of the mode stirrers on the spatial distribution of electric field inside the multimode cavity and, therefore, to infer a mean behavior by the definition of an average electric field in the irradiated dielectric material. Fig. 5 shows the average electric field distribution computed with (4)-(6) for 32 different stirrer positions (see Table I) compared with the spatial field distribution given by Lambert's law and by a constant electric field. It can be noticed that the predicted behavior is, for this particular case, very similar in both cases, with higher electric field values at the edges of the sample.

Likewise, from Fig. 5, one can deduce the assumption of an average constant electric field distribution along the sample would lead to great errors both at the edges and at the center of the sample. On the other hand, Lambert's law cannot properly

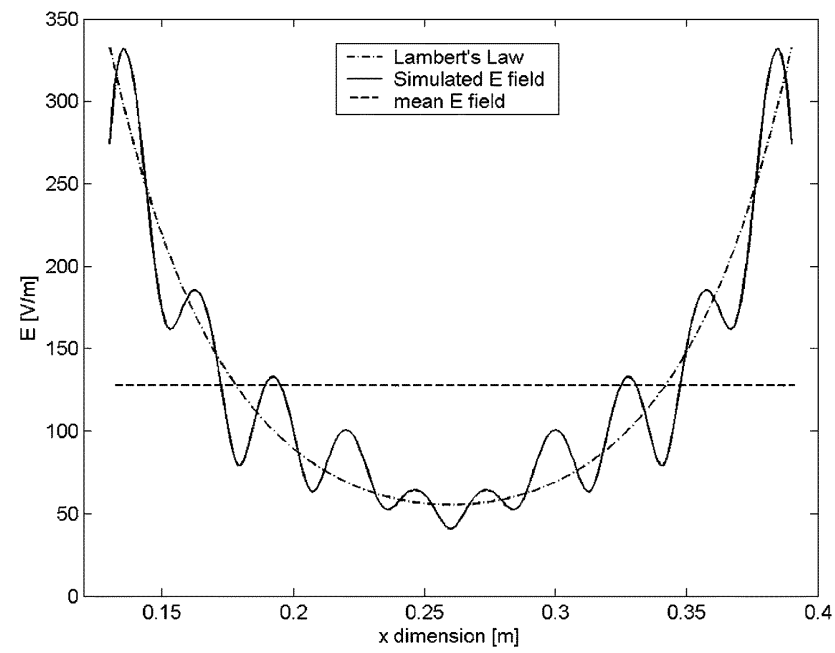

Fig. 5. Comparison of simulated electric field distributions in the sample versus Lambert's law and constant electric field. Sample permittivity is $\varepsilon^{\prime}=30, \varepsilon^{\prime \prime}=5$, and sample thickness $t=10 \mathrm{~mm}$.

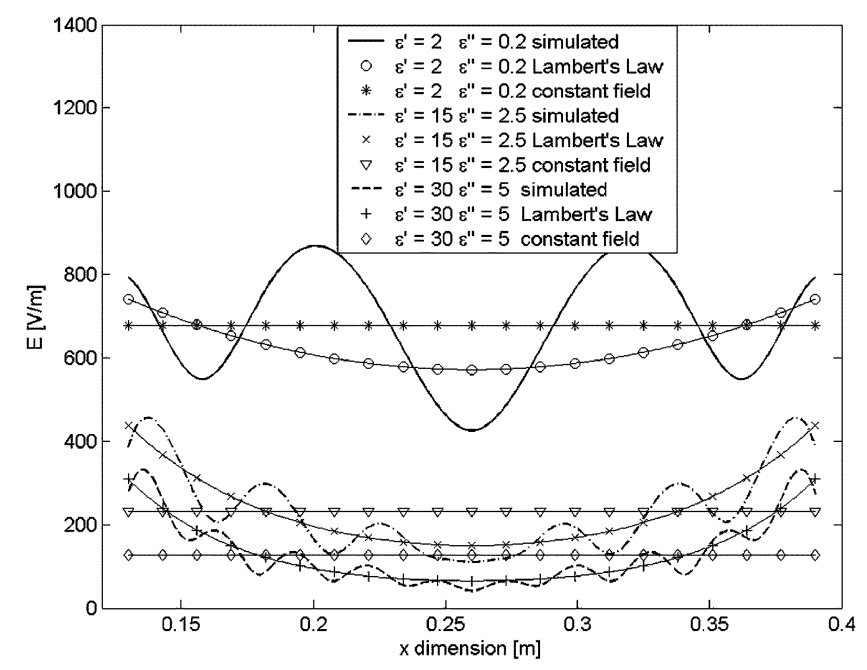

Fig. 6. Comparison of simulated electric field distributions in the material in the microwave applicator as a function of the sample permittivity. Sample thickness is $t=10 \mathrm{~mm}$.

predict the variations of the electric field along the $x$ axis (associated with stationary waves inside the material) providing a mean behavior of the field within the sample. It must also be outlined that Lambert's law underestimates the electric field values at the edges of the sample. For materials with a temperaturedependent loss factor, these differences can lead to great errors in temperature predictions when applying (3), (7)-(10).

\section{A. Effect of Sample Permittivity}

Equation (5) and the same simulation scenario of Fig. 2 have been used to compute the average electric field for samples with different complex permittivity values. Fig. 6 shows the average electric field distribution for low $\left(\varepsilon^{*}=2-0.2 \mathrm{j}\right)$, medium $\left(\varepsilon^{*}=15-2.5 \mathrm{j}\right)$, and high loss factor dielectric materials $\left(\varepsilon^{*}=\right.$ $30-5 \mathrm{j}$ ) computed at the center of the sample.

From Fig. 6, we can see that the electric field distribution is far from being uniform even for low-loss materials. The results also show that, the lower the permittivity of the sample, the higher 


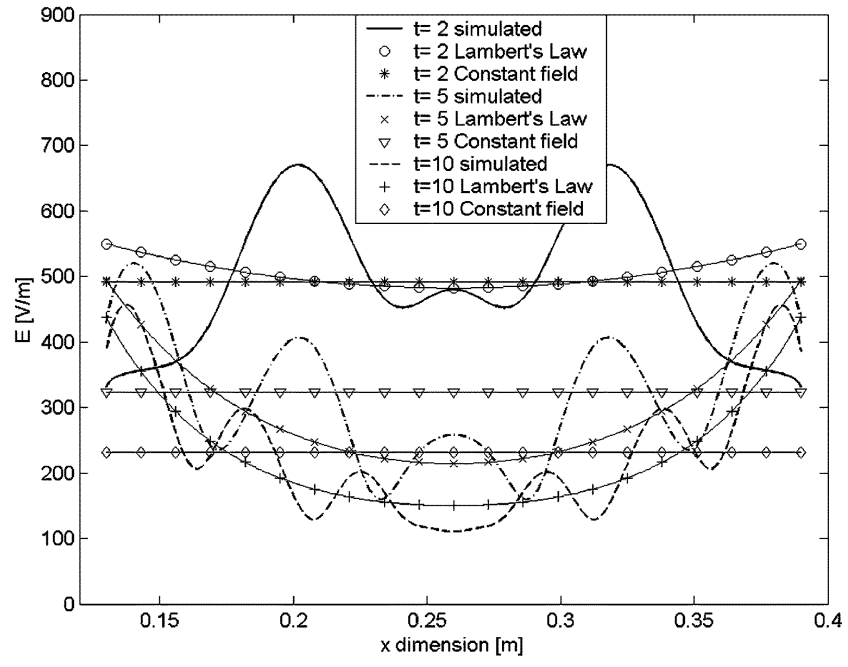

Fig. 7. Comparison of simulated electric field distributions in the material in the microwave applicator for several sample thicknesses $(t)$. Sample permittivity is $\varepsilon^{\prime}=15, \varepsilon^{\prime \prime}=2.5$.

the values of the electric field within the dielectric. It is also clear that, when permittivity decreases, the number of oscillations of electric field are reduced too, mainly due to the increase of wavelength in the material $\left(\lambda_{\varepsilon} \propto \lambda_{\text {atr }} / \sqrt{\varepsilon^{\prime}}\right)$, and that Lambert's law cannot successfully be applied. In fact, Lambert's law seems to be more suitable for materials with high dielectric losses, as for the case of $\varepsilon^{*}=30-5 \mathrm{j}$, than for materials with a low loss factor. On the other hand, the use of a constant electric field distribution does not seem to be a good option whatever the loss factor, due to the fact that these variations of the complex permittivity of the sample force very different boundary conditions and propagation paths that cannot be replaced by uniform electric field distributions.

\section{B. Effect of Sample Geometry}

To study the effect of the geometry on the spatial pattern of the electric field inside the dielectric body, several sample volumes were evaluated. The electric field pattern for three different sample thickness values for two different permittivity values is illustrated in Figs. 7 and 8, respectively.

The curves show that the smaller the thickness of the sample, the higher the electric field inside it and, additionally, that the number of oscillations decrease with decreasing thickness. Thus, the geometry of the sample, together with its permittivity, seems to be very important in the field distribution when studying this type of multimode applicators with laminate stirrers. It is also perceived from Figs. 7 and 8 that the thinner the irradiated material, the bigger the electric field value at the center of the sample. This can be explained because of the lower attenuation of the electric field when using thin materials.

\section{EXPERIMENTAL VALIDATION}

To validate the numerical results, several experimental trials were carried out in a multimode microwave cavity with two laminar stirrers placed at the upper wall, as shown in Fig. 1. The cavity was fed by a magnetron at $2.45 \mathrm{GHz}$ with a nominal output power ranging from 0 to $920 \mathrm{~W}$. The dielectric

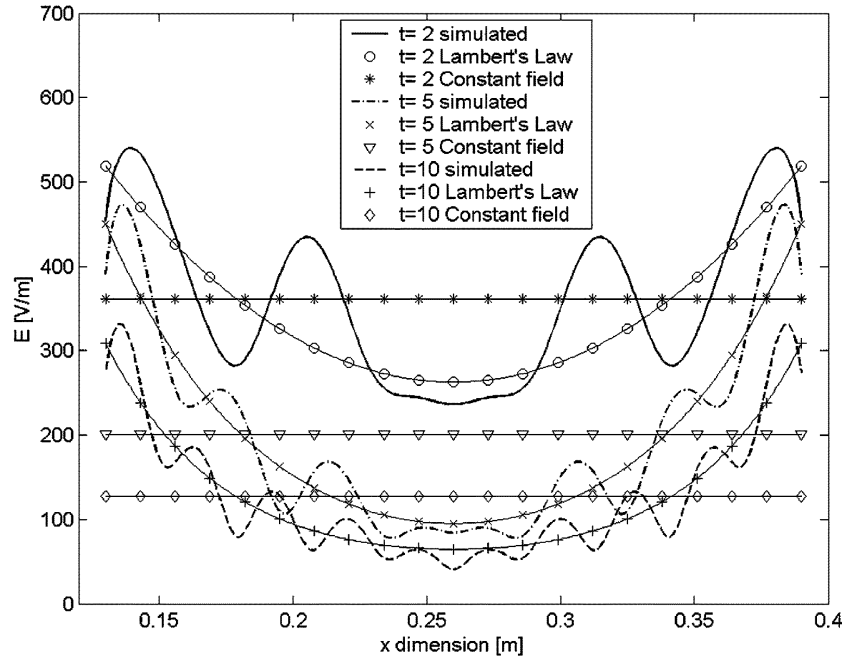

Fig. 8. Comparison of simulated electric field distributions in the material in the microwave applicator for several sample thicknesses $(t)$. Sample permittivity is $\varepsilon^{\prime}=30, \varepsilon^{\prime \prime}=5$.

sample was a 10-mm-thick wet-clay sheet, with an area of $0.26 \times 0.12 \mathrm{~m}^{2}$ and a moisture content around $27 \%$ (dry basis).

The sample support was a thin Teflon grill allowing quasiadiabatic thermal boundary conditions for the sample. To measure the temperature distribution after the irradiation process, an AGEMA VISION thermal camera was used. The measured temperature was used as a validating magnitude due to its direct relationship with the spatial electric field distribution according to (3)-(4). In this way, it was not necessary to use any electric probe inside the applicator, which could introduce inherent perturbations to the electric field distribution.

Dielectric properties of the material employed in the microwave trials were measured with a procedure previously developed in-house [25]. The various clay samples were periodically dehydrated in order to conform a relationship of the dielectric constant and the loss factor with the moisture content. Results for clay relative permittivity values versus moisture content are

$$
\begin{aligned}
\varepsilon^{\prime} & =4.98+88.09 \cdot X \\
\varepsilon^{\prime \prime} & =0.69+42.15 \cdot X
\end{aligned}
$$

where $X$ is the internal moisture content of the clay sample in dry basis.

Fig. 9 shows a thermographic image of the clay sample irradiated in the microwave applicator setup with mode stirrers described in Fig. 1. Microwave power supplied from the generator was around $700 \mathrm{~W}$ and the irradiation time was $1 \mathrm{~min}$. From Fig. 9, we can appreciate that the edges of the sample are heated at a higher rate than its inner parts. This implies that the sample has received a higher concentration of electric field around its perimeter than at the center, which, according to (3)-(4), would lead to higher temperatures. The dotted white line shows the place ( $x$ axis) in which the validation with 2-D simulations was carried out.

Equations (2)-(13) were used to estimate both the average electric field and the temperature distribution in the sample. The parameters for the simulations are listed in Table II. The values 


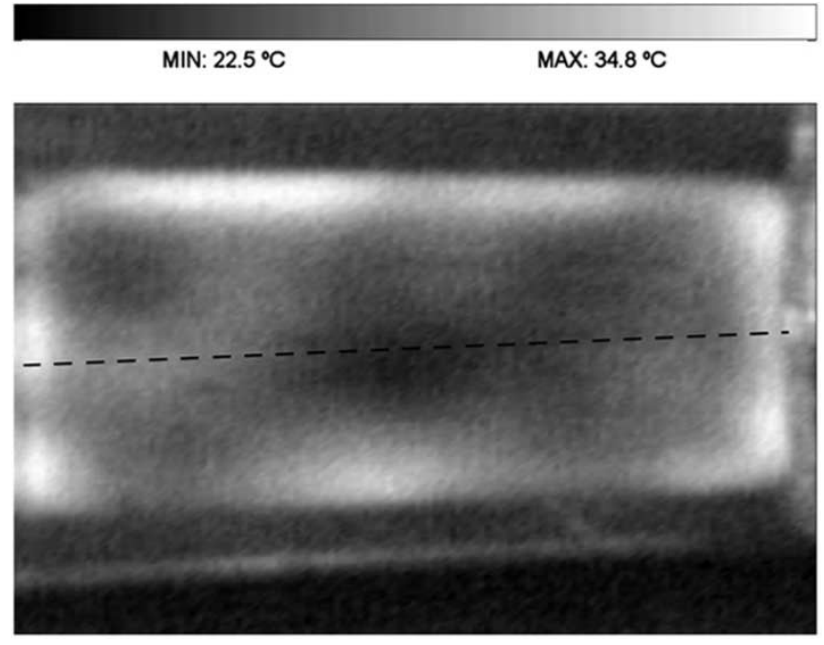

Fig. 9. Thermal image of a wet clay sample after $60 \mathrm{~s}$ of microwave heating in a multimode cavity. Sample dimensions are $0.26 \mathrm{~m}$ ( $x$ axis), $0.01 \mathrm{~m}$ ( $y$ axis), and $0.12 \mathrm{~m}$ ( $z$ axis).

TABLE II

PARAMETERS USED IN SiMULATION FOR Fig. 10

\begin{tabular}{lll}
\hline \hline \multirow{2}{*}{ Symbol } & \multicolumn{1}{c}{ Quantity } & \multicolumn{1}{c}{ Value } \\
\hline$f$ & Frequency & $2.45 \mathrm{GHz}$ \\
$\rho$ & Sample density & $1450 \mathrm{~kg} / \mathrm{m}^{3}$ \\
$c_{p}$ & Sample specific heat & $2378 \mathrm{~J} / \mathrm{kg}$ \\
$k_{t}$ & Sample thermal conductivity & $0.8 \mathrm{~W} / \mathrm{m}^{\circ} \mathrm{C}$ \\
$E_{\text {source }}$ & TE ${ }_{10}$ Electric field peak value & $20780 \mathrm{~V} / \mathrm{m}$ \\
$\varepsilon^{\prime}$ & Sample dielectric constant & 28.77 \\
$\varepsilon^{\prime \prime}$ & Sample loss factor & 12.07 \\
$L$ & Sample Length & $0.26 \mathrm{~m}, \mathrm{x} \in[0.13,0.39] \mathrm{m}$ \\
$t$ & Sample thickness & $10 \mathrm{~mm}, \mathrm{y} \in[0.035,0.045] \mathrm{m}$ \\
& Multimode cavity dimensions & $x=0.52 \mathrm{~m}, y=0.54 \mathrm{~m}$ \\
& Stirrer dimensions & $x=0.01 \mathrm{~m}, y=0.15 \mathrm{~m}$ \\
\hline
\end{tabular}

for the clay dielectric constant, its loss factor, and thermal properties are the corresponding to a moisture content of 0.27 (dry basis). In Table II, $E_{\max }$ represents the maximum amplitude of the $\mathrm{TE}_{10}$ mode in the WR-340 waveguide.

Fig. 10 illustrates the simulated temperature variation for 30and 60-s time intervals at the center of the sample according to conditions of Table II. The results show that despite the mode stirrers' movement, the edges heat at a much higher rate than the center of the sample. This is in good agreement with the experimental results of Fig. 9, also plotted in Fig. 10 for comparison purposes. The slight differences for simulated and measured temperature values in Fig. 10 were mainly due to thermal expansion because of the time lapse from the end of the microwave irradiation until the thermographic image was recorded. The fact that the temperature boundary condition is not a perfect adiabatic one and the appearance of some evaporation of the internal moisture content also must be taken into account to explain temperature differences.

\section{CONCLUSION}

In this paper, a new 2-D method for determining the electric field distribution along dielectric materials placed inside multimode microwave applicators with mode stirrers has been

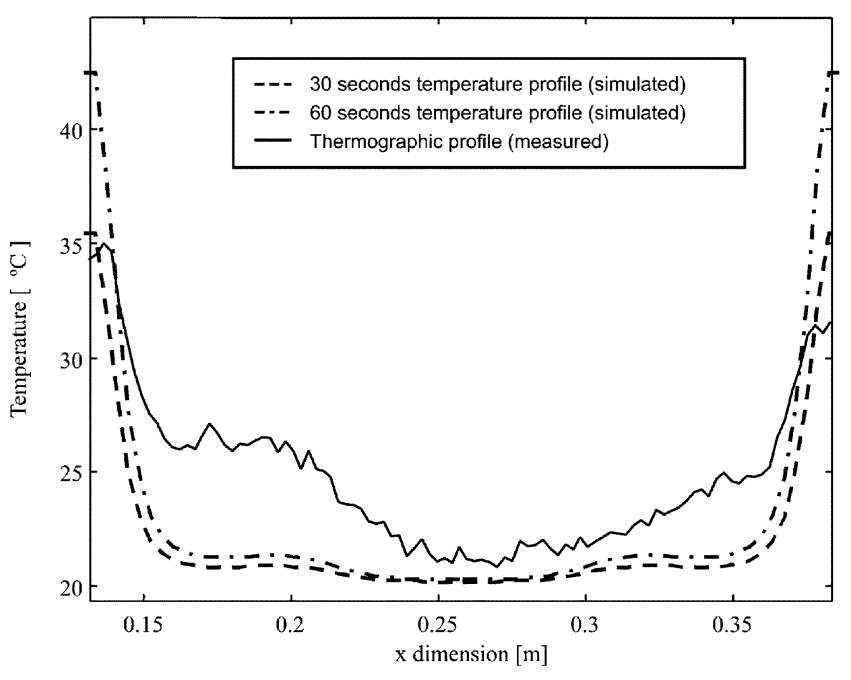

Fig. 10. Comparison between simulated and measured temperature variation along the dielectric sample. Sample positioned from $x=0.13 \mathrm{~m}$ to $x=$ $1.39 \mathrm{~m}$.

described. The method is based on the linear averaging of the absorbed power in the material for different positions of some laminate mode stirrer, where for each spatial position, the wave equation is solved providing an instantaneous electric field distribution in both the cavity and sample.

Simulation results obtained with this method were compared to classical approaches leading to the conclusion that Lambert's law can only be applied when dielectric losses are high while other approaches are no longer valid unless very thin materials are used. When studying low-loss dielectric materials, for instance, several stationary waves can appear within the dielectric body, which cannot be well predicted through the Lambert's law. The use of a constant electric field distribution has also led to big differences with the proposed method reducing the range of validity to very thin materials. Simulated results also showed that both the permittivity and the geometry of the sample have a strong influence on the field spatial distribution.

Despite the 2-D approximation, the good agreement between simulated and experimental temperature profiles shows the good predicting performance of the proposed method for a sample irradiated with microwaves in multimode applicators. This can be particularly important for microwave-assisted drying processes where the permittivity decreases as the body dehydrates, which must lead to a different treatment of the electric field distribution at the initial and final stages of drying. It has been shown that Lambert's law can be used at the initial drying stages where the material behaves as a high lossy material, but it should not be employed at the last stages where the material can be no longer considered a high loss dielectric. In this way, further research is envisaged to determine a rigorous but practical method for industrial microwave drying applicators.

\section{REFERENCES}

[1] A. C. Metaxas and R. J. Meredith, Industrial Microwave Heating. London, U.K.: Peregrinus, 1983, pp. 71-103.

[2] T. V. Chow-Ting-Chan and H. C. Reader, Understanding Microwave Heating Cavities. London, U.K.: Artech House, 2000, pp. 126-163. 
[3] S. C. Kashyap and W. Wyslouzyl, "Methods for improving heating uniformity of microwave ovens," J. Microw. Power Electromagn. Energy, vol. 12, pp. 223-230, 1997.

[4] J. Monzó-Cabrera, "Estudio del Secado Asistido por Microondas en los Materiales Laminares," Ph.D. dissertation (in Spanish), Polytechnic University of Valencia, Valencia, Spain, 2002.

[5] C. A. Balanis, Advance Engineering Electromagnetics. New York: Wiley, 1989, pp. 352-470.

[6] D. C. Dibben and A. C. Metaxas, "Finite element analysis of multimode cavities with coupled electrical and thermal fields," in Proc. 29th IMPI Microwave Power Symp., Chicago, IL, 1994.

[7] _ - "Finite element time domain analysis of multimode applicators using edge elements," J. Microw. Power Electromagn. Energy, vol. 29, pp. 242-251, 1994.

[8] W. Fu and A. C. Metaxas, "Numerical prediction of three-dimensional power density distribution in a multi-mode cavity," J. Microw. Power Electromagn. Energy, vol. 29, pp. 65-77, 1994.

[9] D. C. Dibben and A. C. Metaxas, "Time domain finite element analysis of multimode applicators using edge elements," IEEE Trans. Magn., vol. 32, pp. 942-945, May 1996.

[10] -, "Frequency domain vs. time domain finite element methods for calculations of fields in multimode cavities," IEEE Trans. Magn., vol. 33, pp. 1468-1471, Mar. 1997.

[11] X. Jia, "Experimental and numerical study of microwave power distributions in a microwave heating applicator," J. Microw. Power Electromagn. Energy, vol. 28, pp. 25-31, 1993.

[12] M. Iskander, R. L. Smith, A. O. M. Andrade, H. Kimrey, Jr., and L. M. Wal, "FDTD simulation of microwave sintering of ceramics in multimode cavities," IEEE Trans. Microwave Theory Tech., vol. 42, pp. 793-800, May 1994.

[13] M. Sundberg, P. O. Risman, P.-S. Kildal, and T. Ohlsson, "Analysis and design of industrial microwave ovens using the finite difference time domain method," J. Microw. Power Electromagn. Energy, vol. 31, pp. 142-157, 1996.

[14] P. O. Risman and T. Ohlsson, "Metal in the microwave oven," Microwave World, vol. 13, pp. 28-33, 1992.

[15] K. S. Yee, "Numerical solution of initial boundary value problems involving Maxwell's equations in isotropic media," IEEE Trans. Antennas Propagat., vol. MTT-14, pp. 302-307, 1966.

[16] F. Liu, I. Tumer, and M. E. Bialkowski, "A finite-difference time-domain simulation of power density distribution in a dielectric loaded microwave cavity," J. Microw. Power Electromagn. Energy, vol. 29, pp. 138-148, 1994

[17] L. Ma, D.-L. Paul, N. Pothecary, C. Railton, J. Bows, L. Barratt, J. Mullin, and D. Simons, "Experimental validation of a combined electromagnetic and thermal FDTD model of a microwave heating process," IEEE Trans. Microwave Theory Tech., vol. 43, pp. 2565-2572, Nov. 1995.

[18] D. H. Choi and W. J. R. Hoefer, "The finite-difference-time-domain method and its application to eigenvalue problems," IEEE Trans. Microwave Theory Tech., vol. MTT-34, pp. 1464-1470, Dec. 1986.

[19] A. Taflove and S. C. Hagness, Computational Electrodynamics: The Finite-Difference Time-Domain Method, 2nd ed. Norwood, MA: Artech House, 1995, pp. 67-106.

[20] J. Monzó-Cabrera, A. Díaz-Morcillo, J. M. Catalá-Civera, and D. Sánchez-Hernández, "Study and design of laminar mode stirrers in multimode microwave heating ovens," in Proc. Eur. Symp. Numerical Methods in Electromagnetics (JEE' '02), Mar. 2002.

[21] P. Chen and P. S. Schmidt, "An integral model for drying hygroscopic and nonhygroscopic materials with dielectric heating," Drying Technol., vol. 8, no. 1990, pp. 907-930.

[22] L. Zhou, V. M. Puri, and R. C. Anantheswaran, "Effect of temperature gradient on moisture migration during microwave heating," Drying Technol., vol. 12, pp. 777-798, 1994.

[23] M. A. M. Khraisheh, T. J. R. Cooper, and T. R. A. Mageee, "Microwave and air drying I: Fundamental considerations and assumptions for the simplified thermal calculations of volumetric power absorption," J. Food Eng., vol. 33, pp. 207-219, 1997.

[24] Partial Differential Equation Toolbox. MATLAB. [Online]. Available: http://www.mathworks.com

[25] J. M. Catalá-Civera, A. J. Canós, F. Peñaranda-Foix, and E. de los Reyes, "Accurate determination of the complex permittivity of materials with transmission reflection measurements in partially filled rectangular waveguides," IEEE Trans. Microwave Theory Tech., vol. 50, pp. 16-24, Jan. 2003
Pedro Plaza-González was born in Elda, Spain, in June 1971. He received the Dipl.Ing. degree in telecommunications engineering from the Universidad Politécnica de Valencia, Valencia, Spain, in 1998 . He is currently working toward the Ph.D. degree there.

Since 1999, he has been a Research Assistant with the Microwave Heating Group (GCM) at the Universidad Politécnica de Valencia. His current research areas are focused on microwave-assisted heating and drying processes, microwave power instrumentation, and temperature control in microwave power systems.

Juan Monzó-Cabrera was born in Elda, Spain, in January 1973. He received the Dipl.Ing. and Ph.D. degrees in telecommunications engineering from the Universidad Politécnica de Valencia, Valencia, Spain, in 1998 and 2002, respectively.

From 1999 to 2000, he was a Research Assistant with the Microwave Heating Group (GCM), Universidad Politécnica de Valencia. In 2000, he joined the Departamento de Teoría de la Señal y Radiocomunicaciones, Universidad Politécnica de Cartagena, Cartagena, Spain, as an Associate Lecturer. His current research areas cover microwave-assisted heating and drying processes, microwave applicator design and optimization, and numerical techniques in electromagnetics.

José M. Catalá-Civera was born in Valencia, Spain, on February 15, 1969. He received the Dipl.Ing. and $\mathrm{Ph} . \mathrm{D}$. degrees in telecommunications engineering from the Universidad Politécnica de Valencia, Valencia, Spain, in 1993 and 2000, respectively.

From 1993 to 1996, he was a Research Assistant in the Microwave Laboratory, Universidad Politécnica de Valencia, where he developed several prototypes for industrial applications of microwave engineering. Since 1996, he has been with the Communications Department, Universidad Politecnica de Valencia, where he is involved with research and is also an Associate Lecturer, currently responsible for the Microwave Laboratory. His research interests encompass all aspects related to microwave theory and applications, the use of microwaves for dielectric heating, microwave filters, microwave resonators, methods of dielectric properties measurements, and development of microwave sensors of nondestructive testing of materials.

David Sánchez-Hernández (M’00) received the Dipl.Ing. degree in telecommunications engineering from Universidad Politécnica de Valencia, Valencia, Spain, in 1992 and the Ph.D. degree from King's College, University of London, London, U.K., in 1996.

From 1992 to 1994, he was a Research Associate for The British Council-CAM at King's College London, where he worked on active and dual-band microstrip patch antennas. In 1994, he was appointed EU Research Fellow at King's College London, working on several joint projects at 18, 38, and $60 \mathrm{GHz}$ related to printed and integrated antennas on GaAs, microstrip antenna arrays, sectorization, and diversity. In 1997, he returned to Universidad Politécnica de Valencia, where was co-leader of the Antennas, Microwaves and Radar Research Group and the Microwave Heating Group. In early 1999, he received the Readership from Universidad Politécnica de Cartagena, and was appointed Vice-Dean of the School for Telecommunications Engineering and leader of the Microwave, Radiocommunications and Electromagnetism Engineering Research Group. In late 1999, he was appointed Vice-Chancellor for Innovation and Technology Transfer at Universidad Politécnica de Cartagena and member of several Foundations and Societies for promotion of research and development in the Autonomous Region of Murcia, Spain. In May 2001, he was appointed official advisor in technology transfer and member of The Industrial Advisory Council of the Autonomous Government of the Region of Murcia. He has published over 50 scientific papers and is a reviewer of several international journals. His current research interests encompass all aspects of the design and application of printed antennas and MMICs for mobile communications, microwave heating, and electromagnetic dosimetry issues.

Dr. Sánchez-Hernández is the recipient of the R\&D J. Langham Thompson Premium, awarded by the Institution of Electrical Engineers (IEE). He is an IEE Chartered Engineer (Stage 1), a member of IEE, a member of the Ampere Board, and a member of Cenelec TC106X. 